\title{
The effects of contralateral noise on reaction time to monaural stimuli
}

\author{
PETER G. AITKEN \\ State University of New York College at Brockport, Brockport, New York 14420
}

\begin{abstract}
Experiment I measured reaction time (RT) to monaural tones of six frequencies presented along with white noise to the contralateral ear. RT with the hand ipsilateral to the stimulus was an average of $9.63 \mathrm{msec}$ faster than RT with the contralateral hand. Contralateral RT was significantly affected by the stimulus frequency. Experiment II measured ipsilateral and contralateral RT to monaural tones with and without contralateral noise. Noise-on results agreed with the results of Experiment I, while noise-off results showed no difference between ipsilateral and contralateral RT. No right-ear advantage was found. The ipsilateral-contralateral RT difference found with noise on is interpreted as being due to callosal transmission time as well as other factors. The finding of no right-ear advantage is discussed in relation to other studies which did report a right-ear advantage.
\end{abstract}

The contralateral cortical projections of the visual fields has provided the basis for a number of studies which used reaction time (RT) measurements in attempts to determine the time required for information to be transmitted between the hemispheres of the human brain. Utilizing either the localization of verbal function in the left hemisphere, or the contralateral cortical control of finger movements, these studies compared crossed and uncrossed verbal RT (Filbey \& Gazzaniga, 1969; Moscovitch \& Catlin, 1970) or finger RT (Berlucchi, Heron, Hyman, Rizzolatti, \& Umilta, 1971; Jeeves, $1965)$ to lateralized visual stimuli. Uncrossed reactions, where input and output involve the same hemisphere, do not require interhemispheric transmission of information, while crossed reactions, where input and output involve different hemispheres, do require interhemispheric transmission of information. The time by which crossed reactions have been found to be slower than uncrossed reactions has been interpreted as being due, at least in part, to the time required for information to be transmitted between the hemispheres.

While it is well established that crossed reactions are slower than uncrossed, reports on the magnitude of the difference vary: e.g., $30 \mathrm{msec}$ (Filbey \& Gazzaniga, 1969). $10 \mathrm{msec}$ (Moscovitch \& Catlin, 1970), and $2.7 \mathrm{msec}$ (Berlucchi et al., 1971).

These results, obtained with visual stimulation, might not be predicted in similar studies using auditory stimulation because neural inputs from each ear project to the auditory areas of both

This report is based on a thesis directed by Sachio Ashida and submitted in partial fulfillment of the requirements for the MA degree at the State University of New York, College at Brockport. The author is presently at the University of Connecticut. Reprint requests should be sent to Peter G. Aitken, Department of Psychology, U-20, University of Connecticut, Storrs, Connecticut 06268. hemispheres. Simon (1967) found no difference between crossed and uncrossed RT to monaural auditory stimuli. Using measures other than simple RT, however, a number of investigators have found an ear asymmetry effect. The right ear has typically been found to be superior for language-related sounds (Borkowski. Spreen, \& Stutz, 1965; Bryden, 1963; Curry \& Rutherford, 1967; Dirks, 1964; Gerber \& Goldman. 1971; Kimura, 1961a, b, 1964, 1967; Kimura \& Folb, 1968; Sparks, Goodglass, \& Nickel, 1970; Springer, 1971, 1973; Studdert-Kennedy \& Shankweiler, 1970), and the left ear for certain nonlanguage sounds (Chaney \& Webster, 1966; Curry, 1967; Kimura, 1964; Shankweiler, 1966; Spellacy, 1970). Since, in most people, the left hemisphere is dominant for the processing of language-related input, and the right hemisphere is dominant for the processing of certain nonlanguage inputs. the finding of an ear asymmetry effect indicated that inputs from each ear are transmitted to the contralateral hemisphere more efficiently than to the ipsilateral hemisphere. As noted by Dirks (1964), the ear asymmetry effect is generally found only under conditions of dichotic stimulation. Kimura (1961a, 1967) and Dirks (1964) have related this to Rosenzweig's (1951) finding (see also Hall \& Goldstein, 1968) that, in cats, there is greater evoked cortical response to contralateral than to ipsilateral auditory stimulation, and that under dichotic stimulation ipsilateral auditory pathways are partially occluded by contralateral ones. The data in humans is not as clear: Majkowski, Bochenck, Bochenck, Knapik-Fijalkowska, and Kopec (1971) have reported that while contralateral cortical responses to monaural stimulation have a shorter latency than ipsilateral responses, there is no overall difference between sides. It seems reasonable to assume. however, that under dichotic stimulation the input first reaching one hemisphere (that from the 
contralateral ear) would be processed more efficiently than would a later-arriving input. Dichotic stimulation, therefore, would result in the auditory pathways functioning, to a degree, as if inputs from each ear projected solely to the contralateral cortex.

The situation is not this simple, however; a number of studies have obtained an ear asymmetry effect using monaural stimulation (Bakker, 1967, 1968, 1969, 1970; Doehring, 1972; Frankfurter \& Honeck, 1973; Fry, 1974; Jarvella \& Herman, 1973; Morais \& Darwin, 1974; Murphy \& Venables, 1970). Failures to obtain an ear asymmetry effect with dichotic stimulation have also been reported (Corsi, 1967; Darwin, Note 1). Dichotic stimulation is clearly neither a necessary nor a sufficient condition for ear asymmetry phenomena, and factors other than purely perceptual appear to be involved. This question is discussed by Morais and Darwin (1974).

Studies which have investigated the ear asymmetry effect by measuring right- and left-hand RT to randomly presented monaural tones have given mixed results. Simon (1967) found a right-ear advantage, but no crossed-uncrossed difference. Haydon and Spellacy (1973) found a right-ear advantage for right-hand responses, but not for left-hand responses. They also found uncrossed reactions an average of $9 \mathrm{msec}$ faster than crossed reactions, but this difference was not analyzed statistically. Provins and Jeeves (1975) found a right-ear advantage for right-handed subjects, but not for left-handed subjects. In addition, uncrossed reactions were reported to be significantly faster than crossed reactions, but again only for right-handed subjects. Their statistical analysis, however, was performed with nonparametric methods, and their data suggest that this difference would have been found to be nonsignificant if a factorial analysis of variance had been used.

The present study investigated the ear asymmetry effect in a simple RT task, using monaural tones both with and without contralateral noise. The effect of stimulus frequency was also investigated.

\section{EXPERIMENT I}

\section{Method}

Subjects. The subjects were 10 male undergraduate students who reported having normal hearing and being right-handed.

Apparatus. White noise was from a Grason-Stadler Model 901B noise generator, and signal tones from a Tektronics Model SG-502 oscillator. Stimuli were switched with a home-built integratedcircuit-timed switch and presented through Koss Model K-6 stereo earphones. RT was measured with a Berkeley Model S54 EPUT meter.

Procedure. The subject was seated in a straight-backed chair at a table in an otherwise empty $5 \times 8 \mathrm{ft}$ sound-attenuating room. Air temperature was $70^{\circ} \mathrm{F}$, and room illumination was subdued (approximately $20 \mathrm{~lx}$ ). Each hand was positioned near a reaction switch, which was operated by holding the switch in (toward the midline) and releasing it upon hearing the signal. The subject was informed whether to react with his right or left hand on a given trial by a small red light above the appropriate switch. The lights did not serve as a warning -one or the other was always on, changing, when needed, immediately following a trial.

Reaction signals were monaurally presented pure sinusoidal tones of $400,800,1,200,1,600,2,000$, and $2,400 \mathrm{cps}, 250 \mathrm{msec}$ duration, and $70 \mathrm{~dB}$ re $20 \mu \mathrm{N} / \mathrm{m}^{2}$. Binaural white noise, also at $70 \mathrm{~dB}$, was onset 1 to $5 \mathrm{sec}$ prior to the reaction signal, and was offset $1 \mathrm{sec}$ after the reaction signal offset. For the duration of the signal tone, the white noise was offset in the channel that carried the tone.

Each subject received 10 trials under each of the 24 experimental conditions ( 2 hands, 2 ears, 6 frequencies). The trials were randomly ordered within each of 10 blocks, each block containing every condition once. The time by which the white noise anticipated the signal was randomly assigned to trials with the restrictions that (a) the $\mathbf{1 0}$ trials of each experimental condition had each duration of anticipation $(1,2,3,4$, and $5 \mathrm{sec})$ assigned twice, and (b) within each block the mean anticipation was between 2.5 and $3.5 \mathrm{sec}$. The intertrial interval was random, between 10 and $20 \mathrm{sec}$, averaging $15 \mathrm{sec}$ within each block. Subjects were given 24 practice trials and 5 min rest after three and six blocks had been completed.

$\mathrm{RT}$ was recorded to the nearest millisecond in an adjoining room. Anticipatory (RT $<100 \mathrm{msec}$ ) and delayed (RT $>250 \mathrm{msec}$ ) responses were discarded, and the trial repeated immediately. Subjects were instructed to use a finger movement, not a wrist movement, to respond. The design of the experiment was explained to them, and it was stressed that the side of stimulation would be random and unrelated to the side of response.

\section{Results}

The results of Experiment $I$ are summarized in Figure 1. Each data point represents the mean of 100 responses, 10 by each subject under that condition. The data were averaged across trials and analyzed in a two-factor repeated measures ANOVA. Examination of the data revealed no significant difference between the two crossed response conditions or between the two uncrossed response conditions, permitting the hand and ear factors to be collapsed into a crossed vs. uncrossed factor. Uncrossed reactions were an average of $9.63 \mathrm{msec}$ faster than crossed reactions, a highly significant difference.

Analysis of simple effects are as follows: crossed reactions varied significantly as a function of.

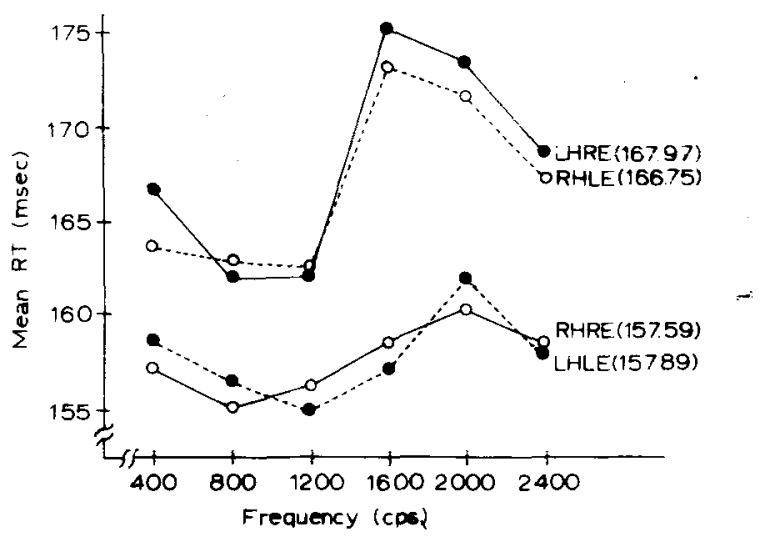

Figure 1. The results of Experiment I. Legend: LHLE means left hand, left ear, etc. The numbers in parentheses are the overall mean RT for each category of response. 
Table 1

Analysis of Variance: Experiment I

\begin{tabular}{lrrr}
\multicolumn{1}{c}{ Source } & df & \multicolumn{1}{c}{ MS } & \multicolumn{1}{c}{ F } \\
\hline Crossed or Uncrossed $(C)$ & 1 & 2776.52 & $180.17^{* *}$ \\
Frequency (F) & 5 & 217.44 & $11.02^{* *}$ \\
Subjects (S) & 9 & 2163.06 & \\
C by F & 5 & 69.68 & $7.13^{* *}$ \\
C by S & 9 & 15.41 & \\
F by S & 45 & 19.73 & \\
C by F by S & 45 & 9.76 & \\
\hline
\end{tabular}

$* * p<.001$

frequency, $F(5,45)=12.50, \mathrm{p}<.001$, while uncrossed reactions did not, $F(5,45)=2.04$, n.s. Crossed reactions were significantly slower than uncrossed reactions at all frequencies, all Fs $(1,9)>$ $14.8, \mathrm{p}<.005$.

\section{EXPERIMENT II}

The crossed-uncrossed.RT difference found in Experiment I could not be properly interpreted until it was determined how much, if any, of this difference was the result of the white noise. In addition to answering this question, Experiment II provided a replication of Experiment $I$ at a single frequency. Reaction signals at $1,600 \mathrm{cps}$ were used in Experiment II because it was at this frequency that the greatest crossed-uncrossed difference was obtained in Experiment $I$.

\section{Method}

Subjects. The subjects were 10 male undergraduate students who reported having normal hearing and being right-handed (not the same individuals as used in Experiment I).

Apparatus. The apparatus were the same as used in Experiment I.

Procedure. The procedure used was the same as used in Experiment I with the following differences. Only one stimulus frequency, $1,600 \mathrm{cps}$, was used. White noise-on trials were as in Experiment 1. Noise-off trials consisted of a 1-sec pulse of binaural white noise, followed by a 1- to 5-sec delay and the signal tone. Each subject received 10 trials under each of the eight experimental conditions (noise on or off, 2 hands, 2 ears). The trials were randomly ordered within each of five blocks, each block containing each condition twice. Intertrial intervals and noise to signal delays were assigned as in Experiment 1 . Twenty-four practice trials were given, and the 80 trials run without interruption.

\section{Results}

The results of Experiment II are summarized in Figure 2. Each data point represents the mean of 100 responses. The data were averaged across trials and analyzed in a two-factor repeated measures ANOVA, summarized in Table 2. As in Experiment I, the data allowed the hand and ear factors to be collapsed into one factor. With noise-on, uncrossed reactions were an average of $13.57 \mathrm{msec}$ faster than crossed reactions, compared with a $16.30-\mathrm{msec}$ difference at $1.600 \mathrm{cps}$ in Experiment I.

Analysis of simple effects showed a significant
Table 2

Analysis of Variance: Experiment II

\begin{tabular}{lccc}
\multicolumn{1}{c}{ Source } & df & MS & F \\
\hline Crossed or Uncrossed (C) & 1 & 334.44 & $16.75^{*}$ \\
White Noise (W) & 1 & 495.34 & $15.47^{*}$ \\
Subjects (S) & 9 & 486.06 & \\
C by W & 1 & 605.59 & $93.88^{* *}$ \\
C by S & 9 & 19.96 & \\
W by S & 9 & 32.01 & \\
C by W by S & 9 & 6.45 & \\
\multicolumn{1}{c}{${ }^{*} p<.005$} & & $* * p<.001$ &
\end{tabular}

difference between crossed and uncrossed reactions with noise on, $F(1,9)=46.40, p<.001$, but not with noise off, $F(1,9)<1$. Noise being on or off had a significant effect on crossed reactions, $F(1,9)=$ $34.30, \mathrm{p}<.001$, but not on uncrossed reactions, $F(1,9)<1$.

\section{DISCUSSION}

This study has clearly shown that RT to monaural tones is longer when crossed than when uncrossed if white noise is presented contralaterally, and that crossed and uncrossed RT to monaural tones alone do not differ. Neither noise-on nor noise-off conditions gave any hand or ear advantage. In addition, the frequency of the signal had a significant effect on crossed reactions. These points will be discussed in turn.

At least part of the increased latency of crossed reactions with contralateral noise is probably due to the time required for interhemispheric transmission of information. The values found in the present study are consistent with the results of some studies which used visual stimuli to study hemisphere differences (Moscovitch \& Catlin, 1970; Rizzolatti. Umilta, \& Berlucchi, 1971), but there are also visual studies which reported much smaller latency differences

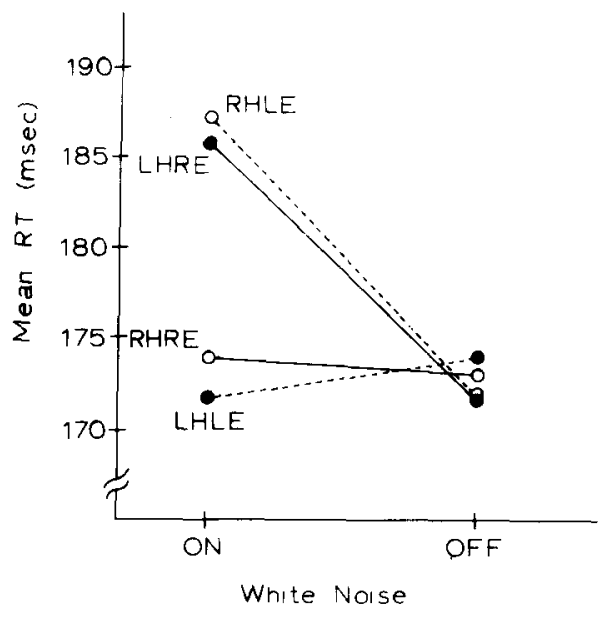

Figure 2. The results of Experiment II. Legend: LHLE means left hand, left ear, etc. 
(Berlucchi et al., 1971; Poffenberger, 1912). If any of these studies were measuring only callosal transmission time, it would have to be those reporting the shorter latency differences. Thus, the crosseduncrossed $\mathrm{RT}$ difference reported here is in all probability due to a combination of callosal transmission time and other, presently unknown, factors.

Stimulus-response compatability effects (Simon, Craft, \& Small, 1970) appear to be absent. Such effects would be expected to influence noise-on and noise-off conditions equally, and the present study found no crossed-uncrossed difference with noise off.

Another possibility might result from subjects having response-side certainty and stimulus-side uncertainty. Readiness to respond on one side might cause involuntary preparation to receive the stimulus on the same side. Such involuntary preparation might affect RT only when detection is difficult due to contralateral white noise. This theory could be tested by repeating Experiment II with both response- and stimulus-side certainty.

There are undoubtedly many factors, other than callosal transmission, which could come into play when the two hemispheres are required to cooperate. Any of these might increase RT, and an unequivocal explanation of the present results obviously must await further experimentation.

The noise-off condition of Experiment II is essentially a duplication of portions of several earlier studies (Haydon \&Spellacy, 1973; Provins \& Jeeves, 1975; Simon, 1967). While these studies gave mixed results, they agree on at least one finding: overall, RT is faster to right-ear stimulation than to left-ear stimulation. The present study found no overall advantage for either ear. Both Haydon and Spellacy (1973) and Simon (1967) explained their results in terms of ear preference or expectancy, while Provins and Jeeves (1975) prefer an explanation " in terms of differential facilitation of neural activity in comparable pathways due to previous usage" (p. 210). The results of Experiment II seem to support the ear-preference or expectancy explanation. The type of neural facilitation suggested by Provins and Jeeves would be expected to be a relatively permanent and universal factor, and should result in a right-ear advantage that is consistently found across both individuals and experiments. Expectancy or preference, on the other hand, might be influenced by specifics of the experimental condition, such as instructions given to the subjects. As the right-ear advantage has not been a consistent phenomenon, across either subjects or experiments, the latter explanation appears more tenable.

The effect of signal frequency on crossed reactions is difficult to interpret. The effect of frequency on uncrossed reactions falls just short of significance $(p<.10)$. Also, examination of Figure 1 shows the two curves to be very similar in shape. The present data do not clearly indicate whether all reactions or only crossed reactions are affected by frequency. Any attempts at explanation should be postponed until this question is answered.

It appears that RT subtraction methods are a valuable tool for the investigation of hemispheric differences. They have been shown, in studies using speech sounds, to give the same results as the more commonly used percent correct scores (Springer, 1971), as well as being able to, in some situations, show differences not demonstrable by percenf correct scores (Springer, 1973). As indicated by the present study, these methods can profitably be extended to stimuli other than speech sounds. A logical continuation of the investigation reported here would be to measure the crossed-uncrossed RT difference as the relative intensity and onset time of the signal and noise are varied. Investigations of this sort may provide further insight into the relationships between the hemispheres.

\section{REFERENCE NOTE}

1. Darwin, C. J. Dichotic forward and backward masking of speech and non-speech sounds. Paper presented to the Acoustical Society of America, Washington, D.C., April 1971.

\section{REFERENCES}

BAKKER, D. J. Left-right differences in auditory perception of verbal and non-verbal material by children. Quarterly Journal of Experimental Psychology, 1967, 19, 334-336.

BAKKER, D. J. Ear-asymmetry with monaural stimulation. Psychonomic Science, 1968, 12, 62.

BAKKER, D. J. Ear-asymmetry with monaural stimulation: Task influences. Cortex, 1969, 5, 36-42.

BAKKER, D. J. Ear-asymmetry with monaural stimulation: Relations to lateral dominance and lateral awareness. Neumopsychologia, 1970, 8, 103-117.

Berlucch, G., Heron, W., Hyman, R., Rizzolatti, G., \& UMilta, C. Simple reaction times of ipsilateral and contralateral hand to lateralized visual stimuli. Brain, 1971, 94, 419-430.

Borkowski, J. G., Spreen, O., \& Stutz, J. F. Ear preference and abstractness in dichotic listening. Psychonomic Science, $1965,3,547-548$.

BRYDEN, M. P. Ear preference in auditory perception. Journal of Experimental Psychology, 1963, 65, 103-105.

Chaney, R. E., \& Webster, J. C. Information in certain multidimensional sounds. Journal of the Acoustical Society of America, 1966, 40, 447-455.

Corsi, P. M. The effects of contralateral noise upon the perception and immediate recall of monaurally presented verbal material. Unpublished MA thesis, McGill University, 1967.

CUrRY, F. K. W. A comparison of left-handed and righthanded subjects on verbal and nonverbal dichotic listening tasks. Cortex, 1967, 3, 343-352.

Curry, F. K. W., \& RUtherford, D. R. Recognition and recall of dichotically presented verbal stimuli by right and left-handed persons. Neuropsychologia, 1967, 5, 119-126.

Dirks, D. Perception of dichotic and monaural verbal material and cerebral dominance for speech. Acta Otolaryngologica, $1964,58,73-80$. 
Doenring, D. G. Ear asymmetry in the discrimination of monaural tone sequences. Canadian Journal of Psychology, 1972, 26, 106-110.

Filbey, R. A., \& Gazzaniga, M. S. Splitting the brain with reaction time. Psychonomic Science, 1969, 17, 335-336.

Frankfurter, A., \& Honeck, R. Ear differences in the recall of monaurally presented sentences. Quarterly Journal of Experimental Psychology, 1973, 25, 138-146.

$F_{R Y}, D$. B. Right ear advantage for speech presented monaurally. Language and Speech, 1974, 17, 142.151.

Gerber, S. E., \& Goldman, P. Ear preference for dichotically presented verbal stimuli as a function of report strategies. Journal of the Acoustical Society of America, 1971, 49, $1163 \cdot 1168$.

HALL, J. L., \& Goldstein, M. H. Representation of binaural stimuli by single units in primary auditory cortex of unanesthetized cats. Journal of the Acoustical Society of America, 1968, 43, 456-461.

Haydon, S. P., \& Spellacy, F. J. Monaural reaction time asymmetries for speech and non-speech sounds. Cortex, 1973. 9. $288-294$.

Jarvella, R. J., \& Herman, S. J. Speed and accuracy of sentence recall: Effects of ear of presentation, semantics, and grammar. Journal of Experimental Psychology, 1973, 97, $108-110$.

JEEves, M. A. Psychological studies of three cases of congenital agenesis of the corpus callosum. Ciba Foundation Study Groups, 1965, 20, 73-94.

Kimura, D. Some effects of temporal lobe damage on auditory perception. Canadian Journal of Psychology, 1961, 15, 156-165. (a)

Kimura, D. Cerebral dominance and the perception of verbal stimuli. Canadian Joumal of Psychology, 1961, 15, 166-171. (b)

Kimura, D. Left-right differences in the perception of melodies. Quarterly Journal of Experimental Psychology, 1964, 16, 355-358.

Kimura, D. Functional asymmetry of the brain in dichotic listening. Cortex, 1967, 3, 163-178.

Kimura, D., \& Folb, S. Neural processing of backwards speech sounds. Science, 1968, 161, 395-396.

Majkowski, J., Bochenck, Z., Bochenck, W., KnapikFijalkowska, D., \& Kopec, J. Latency of averaged evoked potentials to contralateral and ipsilateral auditory stimulation in normal subjects. Bruin Research, 1971, 25, 416-419.

Morais, J., \& DARwin, C. J. Ear differences for same-different reaction times to monaurally presented speech. Brain and Language, 1974, 1, 383-390.
Moscovitch, M., \& Catlin, J. Interhemispheric transmission of information: Measurement in normal man. Psychonomic Science, 1970, 18, 211-213.

Murphy, E. H., \& Venables, P. H. The investigation of ear asymmetry by simple and disjunctive reaction time tasks. Perception \& Psychophysics, 1970, 8, 104-106.

Poffenterger, A. T. Reaction time to retinal stimulation with special reference to time lost in conduction through nerve centers. Archives of Psychology, 1912, 23, 1-73.

Provins, K. A., \& Jeeves, M. A. Hemisphere differences in response time to simple auditory stimuli. Neuropsychologia, $1975,13,207-211$.

Rizzolatti, G., Umilta, C., \& Berlucchi, G. Opposite superiorities of the right and left cerebral hemispheres in discriminative reaction time to physiognomical and alphabetical material. Brain, 1971, 94, 431-442.

Rosenzweic, M. R. Representations of the two ears at the auditory cortex. American Journal of Physiology, 1951, 167, 147-214.

Shankweiler, D. Effects of temporal lobe damage on perception of dichotically presented melodies. Journal of Comparative and Physiological Psychology, 1966, 62, 115-119.

Simon, J. R. Ear preference in a simple reaction time task. Journal of Experimental Psychology, 1967, 75, 49-55.

Simon, J. R., Craft, J. L., \& Small, A. M. Manipulating the strength of a stereotype: Interference effects in an auditory information-processing task. Journal of Experimental Psychology, 1970, 86, 63-68.

Sparks, R., Goodglass, H., \& Nickel, B. Ipsilateral vs. contralateral extinction in dichotic listening resulting from hemispheric lesions. Cortex, 1970, 6, 249-260.

SPELLACY, F. Lateral preferences in the identification of patterned stimuli. Joumal of the Acoustical Society of America, $1970,47,574-578$.

Springer, S. P. Ear asymmetry in a dichotic detection task, Perception \& Psychophysics, 1971, 10, 239-241.

SPRINGer, S. P. Hemispheric specialization for speech opposed by contralateral noise. Perception \& Psychophysics, 1973, 13, 391-393.

Studdert-Kennedy, M., \& Shankweiler, D. Hemispheric specialization for speech perception. Journal of the Acoustical Society of America, $1970,48,579-594$.

(Received for publication August 6, 1975; accepted November 12, 1975.) 